\title{
The Block-Grid Method for Solving Laplace's Equation on Polygons with Nonanalytic Boundary Conditions
}

\author{
A. A. Dosiyev, S. Cival Buranay, and D. Subasi \\ Department of Mathematics, Eastern Mediterranean University, Gazimagusa, Cyprus, Mersin 10, Turkey \\ Correspondence should be addressed to A. A. Dosiyev, adiguzel.dosiyev@emu.edu.tr
}

Received 8 April 2010; Accepted 1 June 2010

Academic Editor: Colin Rogers

Copyright (C) 2010 A. A. Dosiyev et al. This is an open access article distributed under the Creative Commons Attribution License, which permits unrestricted use, distribution, and reproduction in any medium, provided the original work is properly cited.

The block-grid method (see Dosiyev, 2004) for the solution of the Dirichlet problem on polygons, when a boundary function on each side of the boundary is given from $C^{2, \lambda}, 0<\lambda<1$, is analized. In the integral represetations around each singular vertex, which are combined with the uniform grids on "nonsingular" part the boundary conditions are taken into account with the help of integrals of Poisson type for a half-plane. It is proved that the final uniform error is of order $O\left(h^{2}+\varepsilon\right)$, where $\varepsilon$ is the error of the approximation of the mentioned integrals, $h$ is the mesh step. For the $p$-order derivatives $(p=0,1, \ldots)$ of the difference between the approximate and the exact solution in each "singular" part $O\left(\left(h^{2}+\varepsilon\right) r_{j}^{1 / \alpha_{j}-p}\right)$ order is obtained, here $r_{j}$ is the distance from the current point to the vertex in question, $\alpha_{j} \pi$ is the value of the interior angle of the $j$ th vertex. Finally, the method is illustrated by solving the problem in L-shaped polygon, and a high accurate approximation for the stress intensity factor is given.

\section{Introduction}

In the last two decades among different approaches to solve the elliptic boundary value problems with singularities, a special emphasis has been placed on the construction of combined methods, in which differential properties of the solution in different parts of the domain are used (see [1-3], and references therein).

In [3-8], a new combined difference-analytical method called the block-grid method (BGM) is analyzed for the solution of the Laplace equation on polygons, when the boundary functions on the sides causing the singular vertices are given as algebraic polynomials of the arc length. This method is a combination of the exponentially convergent block method (see $[9,10])$ in "singular" part, and the finite difference method, which has a simple structure 
on "nonsingular" part of the polygon. A $k$ th order gluing operator $\left(S^{k}\right)$ is constructed for gluing together the grids and blocks. The uniform estimate for the error of the BGM is of order $O\left(h^{k}\right)$, ( $h$ is the mesh step) when the boundary functions on the sides of the polygon which are not causing the singular vertices are from the Hölder classes $C^{k, \lambda}, 0<\lambda<1$ (see [3-5] for $k=6$, [8] for $k=4$, and [6] for $k=2)$. For the $p$-order derivatives $(p=0,1, \ldots)$ of the difference between the approximate and the exact solutions in each "singular" part, $O\left(h^{2} / r_{j}^{p-1 / \alpha_{j}}\right)$ order is obtained, where $h$ is mesh step, $r_{j}$ is the distance from the current point to the vertex in question, and $\alpha_{j} \pi$ is the value of the interior angle at the considered vertex. Moreover, BGM can give a simple and high accurate formula for the stress intensity factor which is one of the important quantities from an engineering standpoint [11].

In [7] the error of the BGM for the solution of the Dirichlet problem on arbitrary polygons is estimated when the boundary functions on the sides not causing the singular vertices are given from $C^{1,1}$, that is, they have the first derivative, which satisfies a Lipschitz condition. The uniform estimate of order $O\left(h^{2}(|\ln h|+1)\right)$, for the error of the approximate solution, is obtained, and the requirements on the boundary functions cannot be essentially lowered in $C^{k, \lambda}$.

In this paper, the BGM is developed for the solution of Laplace's equation on polygons with nonanalytic boundary conditions of the first kind, that is, we remove the restriction on the boundary functions to be algebraic polynomials on the sides of the polygon causing the singular vertices. It is assumed that the boundary function on each side of the polygon is given from the Hölder classes $C^{2, \lambda}, 0<\lambda<1$. In the integral representation of the solution for each "singular" part, which is combined with the uniform grids on "nonsingular" part, the boundary conditions are taken into account with the help of integrals of Poisson type for a half-plane. Taking $n$ number of quadrature nodes, $n \geq\left[\ln ^{1+\varkappa} h^{-1}\right]+1$, where $\varkappa$ is a fixed number, for the composite midpoint rule in the approximation of the integral representation of the solution, and by evaluating Poisson type integrals with $\varepsilon$ accuracy, the final uniform error is of order $O\left(h^{2}+\varepsilon\right)$. For the $p$-order derivatives $(p=0,1, \ldots)$ of the difference between the approximate and the exact solutions in each "singular" part, $O\left(\left(h^{2}+\varepsilon\right) r_{j}^{1 / \alpha_{j}-p}\right)$ order is obtained. We illustrate the method in solving the problem in L-shaped polygon with the corner singularity, and we give a simple formula for the stress intensity factor for a high accurate approximation.

For the analytical treatment of singularities of a solution of the elliptic equations, see for instance [12-14].

\section{Integral Representation of a Solution}

Let $G$ be an open simply connected polygon, $\gamma_{j}, j=1(1) N$, its sides, including the ends, enumerated counterclockwise, $\gamma=\gamma_{1} \cup \cdots \cup \gamma_{N}$ the boundary of $G$, and $\alpha_{j} \pi, 0<\alpha_{j} \leq 2$, be the interior angle formed by the sides $\gamma_{j-1}$ and $\gamma_{j},\left(\gamma_{0}=\gamma_{N}\right)$. Denote by $A_{j}=\gamma_{j-1} \cap \gamma_{j}$ the vertex of the $j$ th angle, by $r_{j}, \theta_{j}$ a polar system of coordinates with pole in $A_{j}$, where the angle $\theta_{j}$ is taken counterclockwise from the side $\gamma_{j}$.

We consider the boundary value problem

$$
\Delta u=0 \quad \text { on } G, \quad u=\varphi_{j}(s) \text { on } \gamma_{j}, 1 \leq j \leq N
$$

where $\Delta \equiv \partial^{2} / \partial x^{2}+\partial^{2} / \partial y^{2}, \varphi_{j}$ is a given function on $\gamma_{j}$ of the arc length $s$ taken along $\gamma$, and 
$\varphi_{j} \in C^{2, \lambda}\left(\gamma_{j}\right), 0<\lambda<1$, that is, $\varphi_{j}$ has the second derivative on $\gamma_{j}$, which satisfies a Hölder condition with exponent $\lambda$.

At some vertices $A_{j},\left(s=s_{j}\right)$ for $\alpha_{j}=1 / 2$, the continuity condition $\varphi_{j-1}=\varphi_{j}$ is fulfilled. Let $E$ be the set of all $j,(1 \leq j \leq N)$ for which $\alpha_{j} \neq 1 / 2$ or $\alpha_{j}=1 / 2$ but $\varphi_{j-1}\left(s_{j}\right) \neq \varphi_{j}\left(s_{j}\right)$. In the neighborhood of $A_{j}, j \in E$, we construct two fixed block-sectors $T_{j}^{i}=T_{j}\left(r_{j i}\right) \subset G, i=1,2$, where $0<r_{j 2}<r_{j 1}<\min \left\{s_{j+1}-s_{j}, s_{j}-s_{j-1}\right\}, T_{j}(r)=\left\{\left(r_{j}, \theta_{j}\right): 0<r_{j}<r, 0<\theta_{j}<\alpha_{j} \pi\right\}$.

Let (see [15])

$$
\begin{gathered}
\varphi_{j 0}(t)=\varphi_{j}\left(s_{j}+t\right)-\varphi_{j}\left(s_{j}\right), \quad \varphi_{j 1}(t)=\varphi_{j-1}\left(s_{j}-t\right)-\varphi_{j-1}\left(s_{j}\right), \\
Q_{j}\left(r_{j}, \theta_{j}\right)=\varphi_{j}\left(s_{j}\right)+\frac{\left(\varphi_{j-1}\left(s_{j}\right)-\varphi_{j}\left(s_{j}\right)\right) \theta_{j}}{\alpha_{j} \pi}+\frac{1}{\pi} \sum_{k=0}^{1} \int_{0}^{\sigma_{j k}} \frac{\tilde{y}_{j} \varphi_{j k}\left(t^{\alpha_{j}}\right) d t}{\left(t-(-1)^{k} \tilde{x}_{j}\right)^{2}+\tilde{y}_{j}^{2}}
\end{gathered}
$$

where

$$
\begin{gathered}
\tilde{x}_{j}=r_{j}^{1 / \alpha_{j}} \cos \left(\frac{\theta_{j}}{\alpha_{j}}\right), \quad \tilde{y}_{j}=r_{j}^{1 / \alpha_{j}} \sin \left(\frac{\theta_{j}}{\alpha_{j}}\right), \\
\sigma_{j k}=\left|s_{j+1-k}-s_{j-k}\right|^{1 / \alpha_{j}} .
\end{gathered}
$$

It can be shown that the function $Q_{j}\left(r_{j}, \theta_{j}\right)$ has the next properties:

(a) $Q_{j}\left(r_{j}, \theta_{j}\right)$ is harmonic and bounded in the infinite angle $0<r_{j}<\infty, 0<\theta_{j}<\alpha_{j} \pi$;

(b) it satisfies the boundary conditions in (2.1) on $\gamma_{j-1} \cap \bar{T}_{j}^{1}$ and $\gamma_{j} \cap \bar{T}_{j}^{1}, j \in E$, except for the point $A_{j}$ (the vertex of the sector) when $\varphi_{j-1}\left(s_{j}\right) \neq \varphi_{j}\left(s_{j}\right)$, and except at the endpoints of $\gamma_{j-1}$ and $\gamma_{j}$ located at other vertices.

Remark 2.1. We formally set the value of $Q_{j}\left(r_{j}, \theta_{j}\right)$ and the solution $u$ of problem (2.1) at the vertex $A_{j}$ equal to $\left(\varphi_{j-1}\left(s_{j}\right)+\varphi_{j}\left(s_{j}\right)\right) / 2, j \in E$.

Let

$$
R_{j}(r, \theta, \eta)=\frac{1}{\alpha_{j}} \sum_{k=0}^{1}(-1)^{k} R\left(\left(\frac{r}{r_{j 2}}\right)^{1 / \alpha_{j}}, \frac{\theta}{\alpha_{j}},(-1)^{k} \frac{\eta}{\alpha_{j}}\right), \quad j \in E,
$$

where

$$
R(r, \theta, \eta)=\frac{1-r^{2}}{2 \pi\left(1-2 r \cos (\theta-\eta)+r^{2}\right)}
$$

is the kernel of the Poisson integral for a unit circle. 
Lemma 2.2 (Volkov [10]). The solution $u$ of the boundary value problem (2.1) can be represented on $\bar{T}_{j}^{2} \backslash V_{j}, j \in E$, in the form

$$
u\left(r_{j}, \theta_{j}\right)=Q_{j}\left(r_{j}, \theta_{j}\right)+\int_{0}^{\alpha_{j} \pi} R_{j}\left(r_{j}, \theta_{j}, \eta\right)\left(u\left(r_{j 2}, \eta\right)-Q_{j}\left(r_{j 2}, \eta\right)\right) d \eta
$$

where $V_{j}$ is the curvilinear part of the boundary of $T_{j}^{2}$, and $Q_{j}\left(r_{j}, \theta_{j}\right)$ is the function defined by (2.2).

\section{Description of the Block-Grid Method}

The idea of BGM for the solution of problem (2.1) is as follows. Let $t_{j}$ be a polygonal line which lies on $T_{j}^{2}$ and has a positive distance from the vertex $A_{j}$ and curvilinear boundary $V_{j}$ of $T_{j}^{2}, j \in E$. The set of points $T_{j}^{2}$ from $A_{j}$ up to $t_{j}$ is denoted by $\Pi_{j}^{*}$. In the "nonsingular" part of $G, G^{N S}=G \backslash \Pi_{j}^{*}$, the Laplace equation is approximated by finite difference method. On the grids located on $t_{j}, j \in E$, (part of boundary of $G^{\mathrm{NS}}$ ) as a boundary condition, the integral representation (2.7) is used. The integrals in (2.2) are calculated with the given accuracy $\varepsilon$, and for the integral in (2.7), which contains the unknown value $u\left(r_{j 2}, \eta\right)$ of the exact solution, a composite mid-point rule is used. The values at quadrature nodes and at the grids are connected by simplest linear interpolation or by high accurate gluing operators constructed in $[3,5,16]$. After solving the finite difference problem on "nonsingular" part $G^{\mathrm{NS}}$, as an approximate solution at any point of the "singular" part, $\Pi_{j}^{*}, j \in E$ of $G$ is defined by the same approximation of representation (2.7).

Now, we consider one of the realizations of the above-described construction of the BGM (see also [5]).

In addition to the sectors $T_{j}^{1}$ and $T_{j}^{2}$ (see Section 2) in the neighborhood of each vertex $A_{j}, j \in E$, of the polygon, $G$ we construct two more sectors $T_{j}^{3}$ and $T_{j}^{4}$, where $0<r_{j 4}<r_{j 3}<$ $r_{j 2}, r_{j 3}=\left(r_{j 2}+r_{j 4}\right) / 2$, and $T_{k}^{3} \cap T_{l}^{3}=\emptyset, k \neq l, k, l \in E$, and let $G_{T}=G \backslash\left(\bigcup_{j \in E} T_{j}^{4}\right)$.

Let $\Pi_{k} \subset G_{T}, k=1(1) M,(M<\infty)$ be certain fixed open rectangles with arbitrary orientation, generally speaking, with sides $a_{1 k}$ and $a_{2 k}, a_{1 k} / a_{2 k}$, being rational and $G=$ $\left(\bigcup_{k=1}^{M} \Pi_{k}\right) \cup\left(\bigcup_{j \in E} T_{j}^{3}\right)$. Let $\eta_{k}$ be the boundary of the rectangle $\Pi_{k}$ and $V_{j}$ the curvilinear part of the boundary of the sector $T_{j}^{2}$, and $t_{k j}=\eta_{k} \cap \bar{T}_{j}^{3}$. The following general requirement is imposed on the arrangement of the rectangles $\Pi_{k}, k=1(1) M$, and sectors $T_{j}^{2}, j \in E$ : any point $P$ lying on $\eta_{k} \cap G_{T}, 1 \leq k \leq M$, or located on $V_{j} \cap G, j \in E$, falls inside at least one of the rectangles $\Pi_{k(p)}, 1 \leq k(p) \leq M$, depending on $P$, and the distance from $P$ to $G_{T} \bigcap \eta_{k(p)}$ is not less than some constant $\varkappa_{0}>0$ independent of $P$.

The quantity $\varkappa_{0}$ is called a depth of gluing of the rectangles $\Pi_{k}, k=1(1) M$. We introduce the parameter $h \in\left(0, \varkappa_{0} / 2\right]$ and define a square grid on $\Pi_{k}, k=1(1) M$, with maximal possible step $h_{k} \leq \min \left\{h, \min \left\{a_{1 k}, a_{2 k}\right\} / 2\right\}$ such that the boundary $\eta_{k}$ lies entirely on the grid lines. Let $\Pi_{k}^{h}$ be the set of grid nodes on $\Pi_{k}, \eta_{k}^{h}$ the set of nodes on $\eta_{k}$, and $\bar{\Pi}_{k}^{h}=$ $\Pi_{k}^{h} \cup \eta_{k}^{h}$. We denote the set of nodes on the closure of $\eta_{k} \cap G_{T}$ by $\eta_{k 0}^{h}$, the set of nodes on $t_{k j}$ by $t_{k j}^{h}$, and the set of remaining nodes on $\eta_{k}$ by $\eta_{k 1}^{h}$. We also introduce the natural number $n$ and the quantities $n(j)=\max \left\{4,\left[\alpha_{j} n\right]\right\}, \beta_{j}=\alpha_{j} \pi / n(j)$, and $\theta_{j}^{q}=(q-1 / 2) \beta_{j}, j \in E, 1 \leq q \leq n(j)$. 
On the arc $V_{j}$ we choose the points $\left(r_{j 2}, \theta_{j}^{q}\right), 1 \leq q \leq n(j)$, and denote the set of these points by $V_{j}^{n}$.

From the estimation (2.29) in [9] follows the existence of the positive constants $n_{0}$ and $\sigma$ such that, for $n \geq n_{0}$,

$$
\max _{\left(r_{j}, \theta_{j}\right) \in \bar{T}_{j}^{3}} \beta_{j} \sum_{q=1}^{n(j)} R_{j}\left(r_{j}, \theta_{j}, \theta_{j}^{q}\right) \leq \sigma<1
$$

Let

$$
\omega^{h, n}=\left(\bigcup_{k=1}^{M} \eta_{k 0}^{h}\right) \cup\left(\bigcup_{j \in E} V_{j}^{n}\right), \quad \bar{G}_{T}^{h, n}=\omega^{h, n} \cup\left(\bigcup_{k=1}^{M} \bar{\Pi}_{k}^{h}\right) .
$$

We define the matching operator $S^{2}$ at each point $P \in \omega^{h, n}$ in the following way. We consider the set of all rectangles $\left\{\Pi_{k}\right\}$ in the intersections of which the point $P$ lies, and we choose one of these rectangles $\Pi_{k(P)}$, part of whose boundary situated in $G^{T}$ is furthest away from $P$. The value $S^{2} u$ at the point $P$ is computed according to the values of the function at the four vertices $P_{k}, k=1,2,3,4$, of the closure of the cell, containing the point $P$ of the grid constructed on $\bar{\Pi}_{k(P)}$, by multilinear interpolation in the directions of the grid lines. Thus, $S^{2} u$ has the expression

$$
S^{2} u \equiv \sum_{\mu=1}^{4} \lambda_{\mu} u_{\mu}
$$

where $u=u(P), u_{\mu}=u\left(P_{\mu}\right)$, and

$$
\lambda_{\mu} \geq 0, \quad \sum_{\mu=1}^{4} \lambda_{\mu}=1
$$

Let

$$
Q_{j}=Q_{j}\left(r_{j}, \theta_{j}\right), \quad Q_{j 2}^{q}=Q_{j}\left(r_{j 2}, \theta_{j}^{q}\right)
$$

The quantities (3.5) are given by (2.2)-(2.4), which contain integrals that usually cannot be computed exactly. Assume that the approximate values $Q_{j}^{\varepsilon}$ and $Q_{j 2}^{q \varepsilon}$ of the quantities in (3.5) are known with accuracy $\varepsilon>0$, that is,

$$
\left|Q_{j}^{\varepsilon}-Q_{j}\right| \leq c_{1} \varepsilon, \quad\left|Q_{j 2}^{q \varepsilon}-Q_{j 2}^{q}\right| \leq c_{1} \varepsilon,
$$

where $j \in E, 1 \leq q \leq n(j)$, and $c_{1}$ is a constant independent of $\varepsilon$. 
Consider the system of linear algebraic equations

$$
\begin{gathered}
u_{h}^{\varepsilon}=A u_{h}^{\varepsilon} \quad \text { on } \Pi_{k^{\prime}}^{h} \\
u_{h}^{\varepsilon}=\varphi_{m} \quad \text { on } \eta_{k 1}^{h} \cap \gamma_{m}, \\
u_{h}^{\varepsilon}\left(r_{j}, \theta_{j}\right)=Q_{j}^{\varepsilon}+\beta_{j} \sum_{q=1}^{n(j)}\left(u_{h}^{\varepsilon}\left(r_{j 2}, \theta_{j}^{q}\right)-Q_{j 2}^{q \varepsilon}\right) R_{j}\left(r_{j}, \theta_{j}, \theta_{j}^{q}\right) \quad \text { on }\left(r_{j}, \theta_{j}\right) \in t_{k j^{\prime}}^{h} \\
u_{h}^{\varepsilon}=S^{2} u_{h}^{\varepsilon} \quad \text { on } \omega^{h, n},
\end{gathered}
$$

where $1 \leq k \leq M, 1 \leq m \leq N, j \in E$;

$$
A u(x, y)=\frac{(u(x+h, y)+u(x-h, y)+u(x, y+h)+u(x, y-h))}{4} .
$$

Definition 3.1. The solution of the system (3.7)-(3.10) is called a numerical solution of problem (2.1) on $\bar{G}_{T}^{h, n}$.

Definition 3.2. The function

$$
U_{h}^{\varepsilon}\left(r_{j}, \theta_{j}\right)=Q_{j}\left(r_{j}, \theta_{j}\right)+\beta_{j} \sum_{q=1}^{n(j)} R_{j}\left(r_{j}, \theta_{j}, \theta_{j}^{q}\right)\left(u_{h}^{\varepsilon}\left(r_{j 2}, \theta_{j}^{q}\right)-Q_{j 2}^{q \varepsilon}\right)
$$

is called an approximate solution of problem (2.1) on the closed block $\bar{T}_{j}^{3}, j \in E$, where $u_{h}^{\varepsilon}\left(r_{j 2}, \theta_{j}^{q}\right), 1 \leq q \leq n(j), j \in E$, is the solution values of the system (3.7)-(3.10) on $V_{j}^{h}$ (at the quadrature nodes).

Theorem 3.3. There is a natural number $n_{0}$ such that, for all $n \geq n_{0}$ and for any $\varepsilon>0$, the system (3.7)-(3.10) has a unique solution.

Proof. The proof is obtained on the basis of maximum principle by taking into account (3.1), (3.3), (3.4), and (3.11) by analogy with [5].

\section{Convergence of the Block-Grid Equations on "Nonsingular" Part}

Let

$$
\xi_{h}^{\varepsilon}=u_{h}^{\varepsilon}-u,
$$

where $u_{h}^{\varepsilon}$ is a solution of the system (3.7)-(3.10), and $u$ is the trace on $\bar{G}_{T}^{h, n}$ of the solution of (2.1). On the basis of (2.1), (3.7)-(3.10), and (4.1), the error $\xi_{h}^{\varepsilon}$ satisfies the system of difference 
equations

$$
\begin{gathered}
\xi_{h}^{\varepsilon}=A \xi_{h}^{\varepsilon}+r_{h}^{1} \quad \text { on } \Pi_{k^{\prime}}^{h} \\
\xi_{h}^{\varepsilon}=0 \quad \text { on } \eta_{k 1^{\prime}}^{h} \\
\xi_{h}^{\varepsilon}\left(r_{j}, \theta_{j}\right)=\beta_{j} \sum_{q=1}^{n(j)} \xi_{h}^{\varepsilon}\left(r_{j 2}, \theta_{j}^{q}\right) R_{j}\left(r_{j}, \theta_{j}, \theta_{j}^{q}\right)+r_{j h^{\prime}}^{2} \quad\left(r_{j}, \theta_{j}\right) \in t_{k j^{\prime}}^{h} \\
\xi_{h}^{\varepsilon}=S^{2} \xi_{h}^{\varepsilon}+r_{h}^{3} \text { on } \omega^{h, n},
\end{gathered}
$$

where $1 \leq k \leq M, j \in E$,

$$
\begin{gathered}
r_{h}^{1}=A u-u \text { on } \bigcup_{k=1}^{M} \Pi_{k^{\prime}}^{h} \\
r_{j h}^{2}=\beta_{j} \sum_{q=1}^{n(j)}\left(u\left(r_{j 2}, \theta_{j}^{q}\right)-Q_{j 2}^{q \varepsilon}\right) R_{j}\left(r_{j}, \theta_{j}, \theta_{j}^{q}\right)-\left(u-Q_{j}^{\varepsilon}\right) \text { on } \bigcup_{k=1}^{M}\left(\bigcup_{j \in E} t_{k j}^{h}\right), \\
r_{h}^{3}=S^{2} u-u \text { on } \omega^{h, n} .
\end{gathered}
$$

In what follows, for simplicity, we will denote constants which are independent of $h$ and $\varepsilon$ by $c$.

Lemma 4.1. There exists a natural number $n_{0}$ such that, for all $n \geq \max \left\{n_{0},\left[\ln ^{1+\varkappa} h^{-1}\right]+1\right\}$, and $\varepsilon>0$, where $\varkappa>0$ is a fixed number,

$$
\max _{j \in E}\left|r_{j h}^{2}\right| \leq c\left(h^{2}+\varepsilon\right)
$$

Proof. On the basis of (4.4), Lemma 2.2, and by the virtue of $r_{j 3}=\left(r_{j 2}+r_{j 4}\right) / 2<r_{j 2}, t_{k j}^{h} \in \bar{T}_{j}^{3}$, we have

$$
\begin{aligned}
\left|r_{j h}^{2}\right| \leq & \left|\int_{0}^{\alpha_{j} \pi} R_{j}\left(r_{j}, \theta_{j}, \eta\right)\left(u\left(r_{j 2}, \eta\right)-Q_{j}\left(r_{j 2}, \eta\right)\right) d \eta-\beta_{j} \sum_{q=1}^{n(j)}\left(u\left(r_{j 2}, \theta_{j}^{q}\right)-Q_{j 2}^{q}\right) R_{j}\left(r_{j}, \theta_{j}, \eta\right)\right| \\
& +\left|Q_{j}-Q_{j}^{\varepsilon}\right|+\left|\beta_{j} \sum_{q=1}^{n(j)}\left(Q_{j 2}^{q}-Q_{j 2}^{q \varepsilon}\right) R_{j}\left(r_{j}, \theta_{j}, \eta\right)\right|, \quad 1 \leq k \leq M, j \in E .
\end{aligned}
$$

From this, from [9, Lemma 2.10] and inequalities (3.1) and (3.6), it follows that there exists a natural $n_{0}$ such that for all $n \geq n_{0}$, and for the given $\varepsilon>0$, we obtain

$$
\left|r_{j h}^{2}\right| \leq c_{j}^{0} \exp \left\{-d_{j}^{0} n\right\}+c_{1} \varepsilon+c_{1} \sigma \varepsilon, \quad 0<\sigma<1, j \in E,
$$


where $c_{j}^{0}$ and $d_{j}^{0}>0$ are constants, independent of $n$. Putting $c^{0}=\max _{j \in E}\left\{c_{j}^{0}\right\}$, and $d=$ $\min _{j \in E}\left\{d_{j}^{0}\right\}$ from (4.8), we have

$$
\max _{j \in E}\left|r_{j h}^{2}\right| \leq c^{0} \exp \left\{-d^{0} n\right\}+2 c_{1} \varepsilon
$$

Then, for

$$
n \geq \max \left\{n_{0},\left[\ln ^{1+\varkappa} h^{-1}\right]+1\right\}
$$

where $\varkappa>0$ is a fixed number, we have the inequality (4.6).

Since the set of points $\omega^{h, n}$ are strictly interior points of the polygon $G$, then from (4.5) on the basis of Lemma 3 in Chapter III [17], we obtain

$$
\max _{\omega^{h, n}}\left|r_{h}^{3}\right| \leq c h^{2}
$$

Theorem 4.2. There exists a natural number $n_{0}$ such that for $n \geq \max \left\{n_{0},\left[\ln ^{1+\varkappa} h^{-1}\right]+1\right\}$, where $\varkappa>0$ is a fixed number, and for the given $\varepsilon>0$,

$$
\max _{\overline{\mathrm{G}}_{T}^{h, n}}\left|u_{h}^{\varepsilon}-u\right| \leq c\left(h^{2}+\varepsilon\right)
$$

Proof. Let $v_{h}^{\varepsilon}$ be a solution of the system (4.2) when the functions $r_{h^{\prime}}^{1} r_{j h^{\prime}}^{2}$ and $r_{h}^{3}$ in some rectangular grid $\Pi_{k^{*}}^{h}$ are the same as in (4.3)-(4.5), but are zero in $\bar{G}_{T}^{h, n} \backslash \bar{\Pi}_{k^{*}}^{h}$ Let $t_{k^{*} j}^{h} \neq \emptyset$. It is obvious that

$$
W=\max _{\overline{\mathrm{G}}_{T}^{h, n}}\left|v_{h}^{\varepsilon}\right|=\max _{\bar{\Pi}_{k^{*}}^{h}}\left|v_{h}^{\varepsilon}\right|
$$

We represent the function $v_{h}$ on $\bar{G}_{T}^{h, n}$ in the form

$$
v_{h}^{\varepsilon}=\sum_{\kappa=1}^{4} v_{h, k^{\prime}}^{\varepsilon}
$$


where the functions $v_{h, k^{\prime}}^{\varepsilon}, \kappa=2,3,4$, are defined on $\bar{\Pi}_{k^{*}}^{h}$ as a solution of the system of equations

$$
\begin{gathered}
v_{h, 2}^{\varepsilon}=A v_{h, 2}^{\varepsilon} \quad \text { on } \Pi_{k^{* \prime}}^{h}, \quad v_{h, 2}^{\varepsilon}=0 \quad \text { on } \eta_{k^{*} 1^{\prime}}^{h} \\
v_{h, 2}^{\varepsilon}\left(r_{j}, \theta_{j}\right)=r_{j h^{\prime}}^{2} \quad\left(r_{j}, \theta_{j}\right) \in t_{k^{*} j^{\prime}}^{h} \quad v_{h, 2}^{\varepsilon}=0 \quad \text { on } \omega^{h, n} ; \\
v_{h, 3}^{\varepsilon}=A v_{h, 3}^{\varepsilon} \quad \text { on } \Pi_{k^{*} \prime}^{h} \quad v_{h, 3}^{\varepsilon}=0 \quad \text { on } \eta_{k^{*} 1^{\prime}}^{h} \\
v_{h, 3}^{\varepsilon}\left(r_{j}, \theta_{j}\right)=0, \quad\left(r_{j}, \theta_{j}\right) \in t_{k^{*} j^{\prime}}^{h} \quad v_{h, 3}^{\varepsilon}=r_{h}^{3} \quad \text { on } \omega^{h, n} ; \\
v_{h, 4}^{\varepsilon}=A v_{h, 4}^{\varepsilon}+r_{h}^{1} \quad \text { on } \Pi_{k^{* \prime}}^{h} \quad v_{h, 4}^{\varepsilon}=0 \quad \text { on } \eta_{k^{*} 1^{\prime}}^{h} \\
v_{h, 4}^{\varepsilon}\left(r_{j}, \theta_{j}\right)=0, \quad\left(r_{j}, \theta_{j}\right) \in t_{k^{*} j^{\prime}}^{h} \quad v_{h, 4}^{\varepsilon}=0 \quad \text { on } \omega^{h, n},
\end{gathered}
$$

with

$$
v_{h, k}^{\varepsilon}=0, \quad \kappa=2,3,4 \text { on } \bar{G}_{T}^{h, n} \backslash \bar{\Pi}_{k^{*}}^{h} .
$$

Hence according to (4.14)-(4.18) the function $v_{h, k}^{\varepsilon}$ satisfies the system of equations

$$
\begin{gathered}
v_{h, 1}^{\varepsilon}=A v_{h, 1}^{\varepsilon} \quad \text { on } \Pi_{k^{\prime}}^{h} \quad v_{h, 1}^{\varepsilon}=0 \quad \text { on } \eta_{k 1^{\prime}}^{h} \\
v_{h, 1}^{\varepsilon}\left(r_{j}, \theta_{j}\right)=\beta_{j} \sum_{q=1}^{n(j)} R_{j}\left(r_{j}, \theta_{j}, \theta_{j}^{q}\right) S^{2}\left(\sum_{\kappa=1}^{4} v_{h, k}^{\varepsilon}\left(r_{j 2}, \theta_{j}^{q}\right)\right), \quad\left(r_{j}, \theta_{j}\right) \in t_{k j^{\prime}}^{h} \\
v_{h, 1}^{\varepsilon}=S^{2}\left(\sum_{k=1}^{4} v_{h, k}^{\varepsilon}\right) \quad \text { on } \eta_{k 0}^{h}, 1 \leq k \leq M, j \in E,
\end{gathered}
$$

where the functions $v_{h, k}^{\varepsilon}, \mathcal{K}=2,3,4$, are assumed to be known.

Taking into account (4.6) and (4.11), on the basis of (4.15), (4.16), (4.18), and the principle of maximum, we have

$$
\begin{gathered}
W_{2}=\max _{\bar{G}_{T}^{h, n}}\left|v_{h, 2}^{\varepsilon}\right| \leq c\left(h^{2}+\varepsilon\right), \\
W_{3}=\max _{\bar{G}_{T}^{h, n}}\left|v_{h, 3}^{\varepsilon}\right| \leq c h^{2} .
\end{gathered}
$$

The function $v_{h, 4}^{\varepsilon}$ being a solution of the system (4.17), (4.18) is the error of finite difference solution, with step $h_{k^{*}} \leq h$, of the Dirichlet problem on

$$
\Delta w=0 \quad \text { on } \Pi_{k^{*}}, \quad w=\psi_{k^{*}} \quad \text { on } \eta_{k^{*}},
$$


where

$$
\psi_{k^{*}}= \begin{cases}\varphi_{l} & \text { on } \eta_{k^{*} 1} \cap \gamma_{l}, \\ u & \text { on } \eta_{k^{*} 0},\end{cases}
$$

$u$ is a solution of problem (2.1). It is obvious that a solution of problem (4.22) is unique, and $w \equiv u$ on $\bar{\Pi}_{k^{*}}$. As the boundary of $\Pi_{k^{*}}$ located from the vertices $A_{j}, j \in E$, of the polygon $G$ is the distance exceeding some positive quantity independent of $h, \psi_{k^{*}} \in C^{2, \lambda}\left(\eta_{k^{*}}\right), 0<\lambda<1$, and by the virtue of (4.18) and [18, Theorem 1.1], we obtain

$$
W_{4}=\max _{\overline{\mathrm{G}}_{T}^{h, n}}\left|v_{h, 4}^{\varepsilon}\right|=\max _{\bar{\Pi}_{k^{*}}^{h}}\left|v_{h, 4}^{\varepsilon}\right| \leq c h^{2} .
$$

On the basis of (3.1) and (3.4), the function $v_{h, 1}^{\varepsilon}$ is a unique solution of (4.19) (the functions $v_{h, k}^{\varepsilon}, k=2,3,4$, are assumed to be known). By the gluing condition of the rectangles $\Pi_{k}, k=1,2, \ldots, M$, from (4.19) by maximum principle (see [18]), there exists a real number $\lambda^{*}, 0<\lambda^{*}<1$, independent of $h$, such that for $n \geq \max \left\{n_{0},\left[\ln ^{1+\varkappa} h^{-1}\right]+1\right\}$ and for $\varepsilon>0$, we have

$$
\begin{aligned}
& W_{1}=\max _{\overline{\mathcal{G}}_{T}^{h, n}}\left|v_{h, 1}^{\varepsilon}\right| \\
& \leq \max \left\{\max _{\bigcup_{k=1}^{M} \eta_{k 0}}\left|S^{2}\left(\sum_{i=1}^{4} v_{h, i}^{\varepsilon}\right)\right|, \max _{\left(r_{j}, \theta_{j}\right) \in\left(\bigcup_{j \in E} V_{j}^{n}\right)}\left|S^{2}\left(\sum_{i=1}^{4} v_{h, i}^{\varepsilon}\left(r_{j}, \theta_{j}\right)\right)\right| \max _{\left(r_{j}, \theta_{j}\right) \in t_{k j}} \beta_{j} \sum_{q=1}^{n(j)} R_{j}\left(r_{j}, \theta_{j}, \theta_{j}^{q}\right)\right\} \\
& \leq \lambda^{*} W+\sum_{i=2}^{4} \max _{\bar{G}_{T}^{h, n}}\left|v_{h, i}^{\varepsilon}\right| .
\end{aligned}
$$

From (4.13), (4.14), (4.20), (4.21), (4.24), and (4.25), we obtain

$$
W \leq \lambda^{*} W+c\left(h^{2}+\varepsilon\right), \quad 0<\lambda^{*}<1,
$$

that is,

$$
W=\max _{\bar{G}_{T}^{h, n}}\left|v_{h}^{\varepsilon}\right| \leq c\left(h^{2}+\varepsilon\right) .
$$

In the case when $t_{k^{*} j}^{h} \equiv \emptyset$, the function $v_{h}^{2} \equiv 0$ on $\bar{G}_{T}^{h, n}$ and the inequality (4.27) holds true. 
Since the number of grid rectangles in $\bar{G}_{T}^{h, n}$ is finite, for the solution of (4.2), we have

$$
\max _{\overline{\mathrm{G}}_{T}^{h, n}}\left|\xi_{h}^{\varepsilon}\right| \leq c\left(h^{2}+\varepsilon\right) .
$$

\section{Convergence of the Approximate Solution on "Singular" Part}

We consider the question of convergence of the function $U_{h}^{\varepsilon}\left(r_{j}, \theta_{j}\right)$ defined by the formula (3.12). Taking into account the properties of the functions $Q_{j}\left(r_{j}, \theta_{j}\right), j \in E$, and the fact that $R_{j}\left(r_{j}, 0, \eta\right)=R_{j}\left(r_{j}, \alpha_{j} \pi, \eta\right)=0$, the function $U_{h}^{\varepsilon}\left(r_{j}, \theta_{j}\right)$ is defined on $T_{j}^{*}$, where $r_{j}^{*}=\left(r_{j 2}+\right.$ $\left.r_{j 3}\right) / 2$. Moreover, the function $U_{h}^{\varepsilon}\left(r_{j}, \theta_{j}\right)$ is bounded, harmonic on $T_{j}^{*}$, and continuous up to its boundary, except for the vertex $A_{j}$ when the specified boundary values are discontinuous at $A_{j}$. In addition, on the rectilinear parts of the boundary of $T_{j}^{*}$, except, maybe, the vertex $A_{j}$, function $U_{h}^{\varepsilon}\left(r_{j}, \theta_{j}\right)$ satisfies the boundary conditions defined in (2.1).

Theorem 5.1. There is a natural number $n_{0}$, such that for $n=\max \left\{n_{0},\left[\ln ^{1+\varkappa} h^{-1}\right]+1\right\}, \varkappa>0$ is a fixed number, and for any $\varepsilon>0$, the following inequalities are valid:

$$
\left|\frac{\partial^{p}}{\partial x^{p-q} \partial y^{q}}\left(U_{h}^{\varepsilon}\left(r_{j}, \theta_{j}\right)-u\left(r_{j}, \theta_{j}\right)\right)\right| \leq c_{p}\left(h^{2}+\varepsilon\right) \text { on } \bar{T}_{j}^{3}
$$

for integer $1 / \alpha_{j}$ when $p \geq 1 / \alpha_{j}$;

$$
\left|\frac{\partial^{p}}{\partial x^{p-q} \partial y^{q}}\left(U_{h}^{\varepsilon}\left(r_{j}, \theta_{j}\right)-u\left(r_{j}, \theta_{j}\right)\right)\right| \leq \frac{c_{p}\left(h^{2}+\varepsilon\right)}{r^{p-1 / \alpha_{j}}} \quad \text { on } \bar{T}_{j}^{3}
$$

for any $1 / \alpha_{j}$ if $0 \leq p<1 / \alpha_{j}$;

$$
\left|\frac{\partial^{p}}{\partial x^{p-q} \partial y^{q}}\left(U_{h}^{\varepsilon}\left(r_{j}, \theta_{j}\right)-u\left(r_{j}, \theta_{j}\right)\right)\right| \leq \frac{c_{p}\left(h^{2}+\varepsilon\right)}{r^{p-1 / \alpha_{j}}} \quad \text { on } \bar{T}_{j}^{3} \backslash A_{j},
$$

for noninteger $1 / \alpha_{j}$, when $p>1 / \alpha_{j}$. Everywhere $0 \leq q \leq p$, $u$ is a solution of problem (2.1).

Proof. Since $r_{j}^{*}=\left(r_{j 2}+r_{j 3}\right) / r_{j 2}$, then for $n \geq\left[\ln ^{1+\varkappa} h^{-1}\right]+1, \varkappa>0$ is a fixed number, we have

$$
\begin{aligned}
& \mid \beta_{j} \sum_{q=1}^{n(j)} R_{j}\left(r_{j}, \theta_{j}, \theta_{j}^{q}\right)\left(u\left(r_{j 2}, \theta_{j}^{q}\right)-Q_{j}\left(r_{j}, \theta_{j}^{q}\right)\right) \\
& \quad-\int_{0}^{\alpha_{j} \pi} R_{j}\left(r_{j}, \theta_{j}, \eta\right)\left(u\left(r_{j 2}, \eta\right)-Q_{j}\left(r_{j 2}, \eta\right)\right) d \eta \mid \leq c h^{2} \quad \text { on } \bar{T}_{j}^{*}, j \in E .
\end{aligned}
$$


On the basis of (3.1), (3.6), and Theorem 4.2 for $n \geq \max \left\{n_{0},\left[\ln ^{1+\varkappa} h^{-1}\right]+1\right\}$, and for any $\varepsilon>0$, we obtain

$$
\begin{aligned}
& \left|\beta_{j} \sum_{q=1}^{n(j)} R_{j}\left(r_{j}, \theta_{j}, \theta_{j}^{q}\right)\left[\left(u_{h}^{\varepsilon}\left(r_{j 2}, \theta_{j}^{q}\right)-u\left(r_{j 2}, \theta_{j}^{q}\right)\right)+\left(Q_{j}\left(r_{j}, \theta_{j}^{q}\right)-Q_{j 2}^{q \varepsilon}\right)\right]\right| \\
& \quad \leq c\left(h^{2}+\varepsilon\right) \text { on } \bar{T}_{j}^{*}, j \in E .
\end{aligned}
$$

In accordance with the formulae (2.7), (3.12), (5.4), and (5.5) for all $n \geq$ $\max \left\{n_{0},\left[\ln ^{1+\varkappa} h^{-1}\right]+1\right\}$, for any $\varepsilon>0$, we have

$$
\left|U_{h}^{\varepsilon}\left(r_{j}, \theta_{j}\right)-u\left(r_{j}, \theta_{j}\right)\right| \leq c\left(h^{2}+\varepsilon\right) \quad \text { on } \bar{T}_{j}^{*}, j \in E
$$

Let

$$
\varsigma_{h}^{\varepsilon}\left(r_{j}, \theta_{j}\right)=U_{h}^{\varepsilon}\left(r_{j}, \theta_{j}\right)-u\left(r_{j}, \theta_{j}\right) \quad \text { on } \bar{T}_{j}^{*}, j \in E \text {. }
$$

From (3.12), (5.7), and Remark 2.1, it follows that the function $\varsigma_{h}^{\varepsilon}\left(r_{j}, \theta_{j}\right)$ is continuous on $\bar{T}_{j}^{*}$ and is a solution of the boundary value problem

$$
\begin{gathered}
\Delta S_{h}^{\varepsilon}=0 \quad \text { on } T_{j}^{*}, \\
\varsigma_{h}^{\varepsilon}=0 \quad \text { on } \gamma_{m} \cap \bar{T}_{j}^{*}, m=j-1, j, \\
S_{h}^{\varepsilon}\left(r_{j}^{*}, \theta_{j}\right)=U_{h}^{\varepsilon}\left(r_{j}^{*}, \theta_{j}\right)-u\left(r_{j}^{*}, \theta_{j}\right), \quad 0 \leq \theta_{j} \leq \alpha_{j} \pi,
\end{gathered}
$$

where according to (5.6)

$$
\max _{0 \leq \theta_{j} \leq \alpha_{j} \pi}\left|s_{h}^{\varepsilon}\left(r_{j}^{*}, \theta_{j}\right)\right| \leq c\left(h^{2}+\varepsilon\right)
$$

Taking into account (5.9), from [19, Lemma 3.3] follows all inequalities of Theorem 5.1.

Remark 5.2. The right-hand side of the estimations (4.12) and (5.1)-(5.3) depend on the step size $h$ of the grid, and on the parameter $\varepsilon>0$ determining the accuracy of the approximations of the quantities (3.5) in the formulation of the algebraic equations (3.7)-(3.10). Thus, for simplicity, we can set $h \asymp \varepsilon^{-1 / 2}$.

\section{Schwarz's Alternating Method for the System of Block-Grid Equations}

We define the following classes $B_{q}, q=1,2, \ldots, q^{*}$, of rectangles $\Pi_{k}, k=1,2, \ldots, M$ (see [5]). Class $B_{1}$ includes all rectangles whose intersection with the boundary $\gamma$ of the polygon $G$ 
contains a certain segment of positive length. Class $B_{2}$ contains all the rectangles which are not in the class $B_{1}$, whose intersection with rectangles of $B_{1}$ contains a segment of finite length, and so on.

We calculate the values $Q_{j}^{\varepsilon}\left(r_{j 2}, \theta_{j}^{q}\right)$ for all $j \in E, 1 \leq q \leq n(j)$, and the values $Q_{j}^{\varepsilon}\left(r_{j}, \theta_{j}\right)$ on the grids $t_{j}^{h}, j \in E$, with the given accuracy of $\varepsilon$. Suppose, we have a zero approximation $u_{h}^{\varepsilon(0)}$ to the exact solution $u_{h}^{\varepsilon}$ of (3.7)-(3.10). Finding $u_{h}^{\varepsilon(1)}$ for all $j \in E$ by the formula (3.9) on $t_{j}^{h}$ and on $\eta_{k 0}$ by (3.10), we solve the system (3.7)-(3.10) on each grid $\bar{\Pi}_{k}^{h}$ of rectangles, first from class $B_{1}$, then from class $B_{2}$, and so on. The next iteration is similar.

Consequently, we have the sequence $u_{h}^{\varepsilon(1)}, u_{h}^{\varepsilon(2)}, \ldots$, generated by the Schwarz's alternating method

$$
\begin{gathered}
u_{h}^{\varepsilon(m)}\left(r_{j}, \theta_{j}\right)=Q_{j}^{\varepsilon}\left(r_{j}, \theta_{j}\right)+\beta_{j} \sum_{q=1}^{n(j)} R_{j}\left(r_{j}, \theta_{j}, \theta_{j}^{q}\right)\left(u^{\varepsilon(m-1)}\left(r_{j 2}, \theta_{j}^{q}\right)-Q_{j}^{\varepsilon}\left(r_{j 2}, \theta_{j}^{q}\right)\right) \quad \text { on } t_{j}^{h}, \\
u_{h}^{(m)}=S^{2} u_{h}^{\varepsilon(m-1)} \text { on } \omega^{h, n}, \\
u_{h}^{(m)}=A u_{h}^{\varepsilon(m)} \text { on } \Pi_{k^{\prime}}^{h} \quad u_{h}^{\varepsilon(m)}=\varphi \quad \text { on } \eta_{k 1}^{h},
\end{gathered}
$$

where $1 \leq k \leq M, j \in E, m=1,2, \ldots$

Theorem 6.1. For $n \geq \max \left\{n_{0},\left[\ln ^{1+x} h^{-1}\right]+1\right\}$ and for each $\varepsilon>0$, the system (3.7)-(3.10) can be solved by Schwarz's alternating method with any accuracy $\epsilon>0$ in a uniform metric with the number of iterations $O\left(\ln \epsilon^{-1}\right)$, independent of $h, n$, and $\varepsilon$ where $n_{0}$ and $\varkappa$ mean the same as in Theorem 5.1.

Proof. The proof is obtained by analogy with the proof of Theorem 3 from [5].

Remark 6.2. In the case when on the sides of a sector $T_{j}^{1}, j \in E$, the boundary functions are given by algebraic polynomials of $s$, it is expedient to use a simpler elementary harmonic function as in [5] instead of the function (2.2).

Remark 6.3. From the error estimation formula (5.2) of Theorem 5.1, it follows that the error of the approximate solution on the "singular" parts decreases as $r_{j}^{1 / \alpha_{j}}\left(h^{2}+\varepsilon\right)$, which gives an additional accuracy of the BGM near the "singular" points.

Remark 6.4. The method and results of this paper hold for multiply-connected polygons.

\section{Stress Intensity Factor}

Let, in the condition $\varphi_{j} \in C^{2, \lambda}\left(\gamma_{j}\right)$, the exponent $\lambda$ be such that

$$
\left\{\alpha_{j}(2+\lambda)\right\} \neq 0, \quad\left\{2 \alpha_{j}(2+\lambda)\right\} \neq 0,
$$

where $\{\cdot\}$ is the symbol of fraction part. These conditions for given $\alpha_{j}$ can be fulfilled by decreasing $\lambda$ and letting $n_{\alpha_{j}}=\left[\alpha_{j}(2+\lambda)\right],[\cdot]$ be the integer part. 
On the basis of [20, Section 2], a solution of problem (2.1) can be represented in the form

$$
u\left(x_{j}, y_{j}\right)=\tilde{u}\left(x_{j}, y_{j}\right)+\sum_{k=0}^{2} \mu_{k}^{(j)} \operatorname{Im}\left\{z^{k} \ln z\right\}+\sum_{k=1}^{n_{\alpha}} \tau_{k}^{(i)} r_{j}^{k / \alpha} \sin \frac{k \theta}{\alpha}
$$

where $z=x_{j}+i y_{j}, \mu_{k}^{(j)}$, and $\tau_{k}^{(j)}$ are some numbers, and $\tilde{u}\left(x_{j}, y_{j}\right) \in C_{2, \lambda}\left(\bar{T}_{j}\right)$ is harmonic on $T_{j}$. By taking $\theta_{j}=\alpha_{j} \pi / 2$ from the formula (7.2), it follows that the coefficient $\tau_{1}$ which is called the stress intensity factor can be represented as

$$
\tau_{1}^{(j)}=\lim _{r_{j} \rightarrow 0} \frac{1}{r_{j}^{1 / \alpha_{j}}}\left(u\left(x_{j}, y_{j}\right)-\tilde{u}\left(x_{j}, y_{j}\right)-\sum_{k=0}^{2} \mu_{k}^{(j)} \operatorname{Im}\left\{z^{k} \ln z\right\}\right) .
$$

From the formulae (2.2), (3.12), and (7.3), it follows that $\tau_{1, n}^{(j) \varepsilon}$ can be approximated by

$$
\begin{aligned}
\tau_{1, n}^{(j) \varepsilon} & =\lim _{r_{j} \rightarrow 0} \frac{1}{r_{j}^{1 / \alpha_{j}}}\left(U_{h}^{\varepsilon}\left(r_{j}, \theta_{j}\right)-\left(\varphi_{j}\left(s_{j}\right)+\left(\varphi_{j-1}\left(s_{j}\right)-\varphi_{j}\left(s_{j}\right)\right) \frac{\theta_{j}}{\alpha_{j} \pi}\right)\right) \\
& =\lim _{r_{j} \rightarrow 0} \frac{1}{r_{j}^{1 / \alpha_{j}}} \frac{1}{\pi}\left[\sum_{k=0}^{1} \int_{0}^{\sigma_{j k}} \frac{\tilde{y}_{j} \varphi_{j k}\left(t^{\alpha_{j}}\right) d t}{\left(t-(-1)^{k} \tilde{x}_{j}\right)^{2}+\tilde{y}_{j}^{2}}+\beta_{j} \sum_{q=1}^{n(j)}\left(u_{h}^{\varepsilon}\left(r_{j 2}, \theta_{j}^{q}\right)-Q_{j 2}^{q \varepsilon}\right) R_{j}\left(r_{j}, \theta_{j}, \theta_{j}^{q}\right)\right] .
\end{aligned}
$$

Using the formulae (2.3), (2.5), (2.6) from (7.4) for the stress intensity factor, we obtain the next formula

$$
\begin{aligned}
\tau_{1, n}^{(j) \varepsilon}= & \frac{1}{\pi} \int_{0}^{\sigma_{j 0}} \frac{\varphi_{j 0}\left(t^{\alpha_{j}}\right) d t}{t^{2}+r_{j}^{2 / \alpha_{j}}}+\frac{1}{\pi} \int_{0}^{\sigma_{j 1}} \frac{\varphi_{j 1}\left(t^{\alpha_{j}}\right) d t}{t^{2}+r_{j}^{2 / \alpha_{j}}} \\
& +\frac{2}{n(j) r_{j 2}^{1 / \alpha_{j}}} \sum_{q=1}^{n(j)}\left(u_{h}^{\varepsilon}\left(r_{j 2}, \theta_{j}^{q}\right)-Q_{j 2}^{q \varepsilon}\right) \sin \frac{1}{\alpha_{j}} \theta_{j}^{q} .
\end{aligned}
$$

\section{Numerical Results}

Example 8.1. Let $G$ be L-shaped and defined as follows:

$$
G=\{(x, y):-1<x<1,-1<y<1\} \backslash \Omega,
$$

where $\Omega=\{(x, y): 0 \leq x \leq 1,-1 \leq y \leq 0\}$, and $\gamma$ the boundary of $G$. 


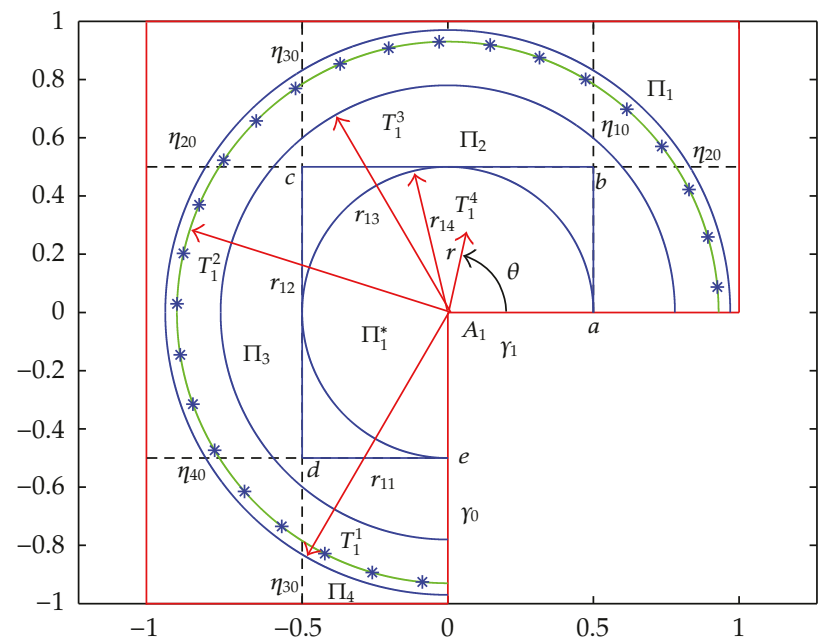

Figure 1: Description of BGM for the L-shaped domain.

We consider the following problem:

$$
\begin{gathered}
\Delta u=0 \text { in } G, \\
u=v(r, \theta) \text { on } r,
\end{gathered}
$$

where

$$
v(r, \theta)=\frac{9}{56} r^{8 / 3} \cos \left(\frac{8}{3} \theta\right)+r^{2 / 3} \sin \left(\frac{2}{3} \theta\right)
$$

is the exact solution of this problem.

We choose a "singular" part of $G$ as

$$
G^{S}=\{(x, y):-0.5<x<0.5,-0.5<y<0.5\} \backslash \Omega_{1},
$$

where $\Omega_{1}=\{(x, y): 0 \leq x \leq 0.5,-0.5 \leq y \leq 0\}$. Then $G^{\mathrm{NS}}=G \backslash G^{S}$ is a "nonsingular" part of $G$.

The given domain $G$ is covered by four overlapping rectangles $\Pi_{k}, k=1, \ldots, 4$, and by the block sector $T_{1}^{3}$ (see Figure 1). For the boundary of $G^{S}$ on $G$, that is, $t_{1}$ the polygonal line abcde is taken. The radius $r_{12}$ of sector $T_{1}^{2}$ is taken as 0.93. According to (8.3), the function $Q(r, \theta)$ in (2.2) is

$$
Q(r, \theta)=\frac{1}{\pi} \frac{9}{56} \int_{0}^{1} \frac{\tilde{y} t^{4} d t}{(t-\tilde{x})^{2}+\tilde{y}^{2}}+\frac{1}{\pi} \frac{9}{56} \int_{0}^{1} \frac{\tilde{y} t^{4} d t}{(t+\tilde{x})^{2}+\tilde{y}^{2}}
$$




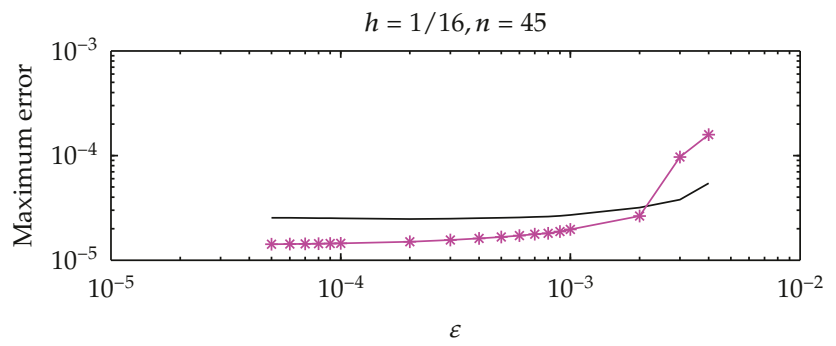

(a)

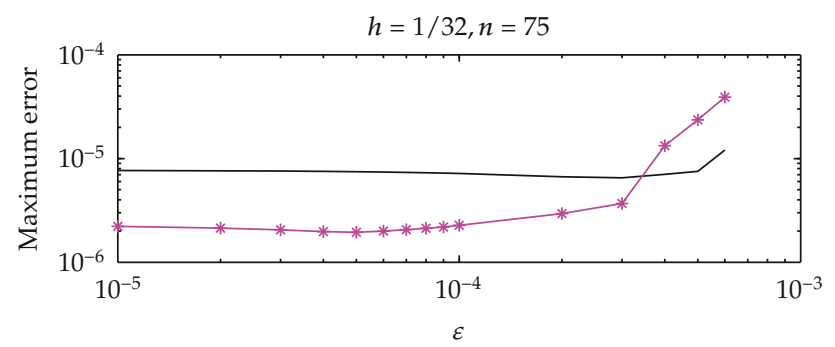

$-\left\|\zeta_{h}\right\|_{\left(G^{\mathrm{NS}}\right)}$

$\rightarrow\left\|\zeta_{h}\right\|_{\left(G^{s}\right)}$

(b)

Figure 2: Dependence on $\varepsilon$ for $h^{-1}=16,32$.

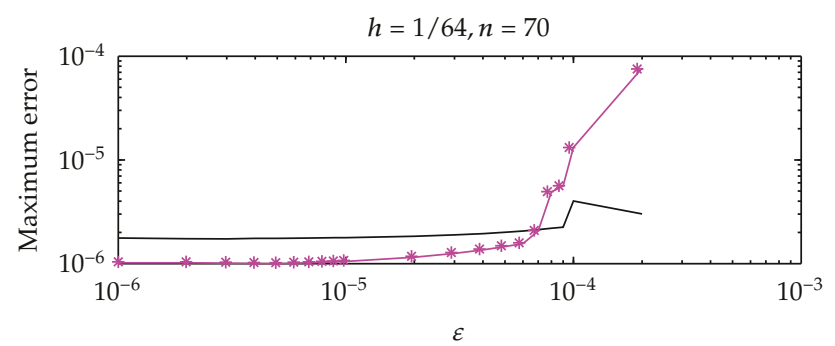

(a)

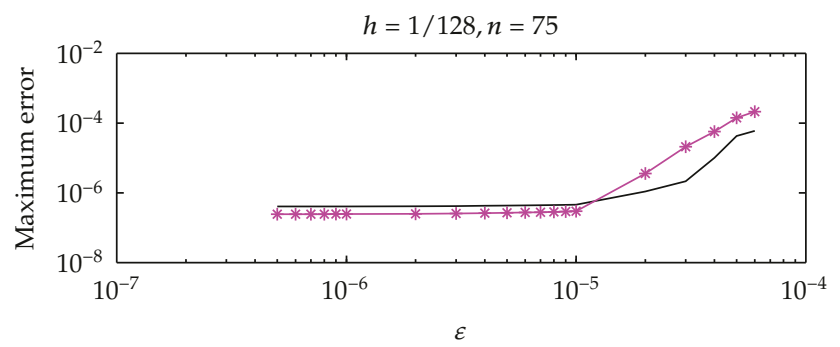

$-\left\|\zeta_{h}\right\|_{\left(G^{N S}\right)}$

$\rightarrow\left\|\zeta_{h}\right\|_{\left(G^{S}\right)}$

(b)

Figure 3: Dependence on $\varepsilon$ for $h^{-1}=64,128$. 
Table 1: The order of convergence in "nonsingular" part when $h=2^{-m}$ and $\varepsilon=5 \times 10^{-9}$.

\begin{tabular}{lcc}
\hline$\left(2^{-m}, n\right)$ & $\left\|\zeta_{h}^{\varepsilon}\right\|_{G^{N S}}$ & $\Re_{G^{N S}}^{m}$ \\
\hline$\left(2^{-4}, 70\right)$ & $3.221892 \times 10^{-5}$ & 3.92 \\
$\left(2^{-5}, 70\right)$ & $8.21629 \times 10^{-6}$ & 4.36 \\
\hline$\left(2^{-5}, 45\right)$ & $9.605851 \times 10^{-6}$ & 3.995 \\
$\left(2^{-6}, 50\right)$ & $2.198578 \times 10^{-6}$ & 4.08 \\
\hline$\left(2^{-5}, 70\right)$ & $8.216298 \times 10^{-6}$ & 3.99 \\
$\left(2^{-6}, 90\right)$ & $2.056572 \times 10^{-6}$ & \\
\hline$\left(2^{-6}, 45\right)$ & $4.244817 \times 10^{-6}$ & \\
$\left(2^{-7}, 85\right)$ & $1.037951 \times 10^{-6}$ & \\
\hline$\left(2^{-6}, 75\right)$ & $2.152420 \times 10^{-6}$ & \\
$\left(2^{-7}, 100\right)$ & $3.387851 \times 10^{-7}$ & \\
\hline
\end{tabular}

Table 2: The order of convergence in "singular" part when $h=2^{-m}$ and $\varepsilon=5 \times 10^{-9}$.

\begin{tabular}{lcr}
\hline$\left(2^{-m}, n\right)$ & $\left\|\zeta_{h}^{\varepsilon}\right\|_{G^{S}}$ & $\mathfrak{R}_{G^{S}}^{m}$ \\
\hline$\left(2^{-4}, 70\right)$ & $1.5957022 \times 10^{-5}$ & 4.37 \\
$\left(2^{-5}, 65\right)$ & $3.6479479 \times 10^{-6}$ & 4.11 \\
\hline$\left(2^{-5}, 45\right)$ & $8.569069 \times 10^{-6}$ & 3.97 \\
$\left(2^{-6}, 50\right)$ & $2.084891 \times 10^{-6}$ & 3.96 \\
\hline$\left(2^{-5}, 65\right)$ & $3.647947 \times 10^{-6}$ & \\
$\left(2^{-6}, 100\right)$ & $9.174034 \times 10^{-7}$ & 4.18 \\
\hline$\left(2^{-6}, 45\right)$ & $4.383277 \times 10^{-6}$ & \\
$\left(2^{-7}, 85\right)$ & $1.105764 \times 10^{-6}$ & \\
\hline$\left(2^{-6}, 85\right)$ & $2.648988 \times 10^{-6}$ & \\
$\left(2^{-7}, 100\right)$ & $6.332288 \times 10^{-7}$ & \\
\hline
\end{tabular}

Table 3: The minimum errors of the solution over the pairs $\left(h^{-1}, n\right)$ in maximum norm when $\varepsilon=5 \times 10^{-9}$.

\begin{tabular}{lccc}
\hline$\left(h^{-1}, n\right)$ & $\left\|\zeta_{h}^{\varepsilon}\right\|_{G^{\mathrm{NS}}}$ & $\left\|\zeta_{h}^{\varepsilon}\right\|_{G^{S}}$ & Iteration \\
\hline$(16,45)$ & $2.567 \times 10^{-5}$ & $1.391 \times 10^{-5}$ & 15 \\
$(32,70)$ & $8.216 \times 10^{-6}$ & $8.732 \times 10^{-6}$ & 16 \\
$(64,70)$ & $1.736 \times 10^{-6}$ & $1.035 \times 10^{-6}$ & 17 \\
$(128,75)$ & $4.038 \times 10^{-7}$ & $2.399 \times 10^{-7}$ & 17 \\
\hline
\end{tabular}

Table 4: The minimum errors of the derivatives over the pairs $\left(h^{-1}, n\right)$ in maximum norm when $\varepsilon=5 \times 10^{-9}$.

\begin{tabular}{lcc}
\hline$\left(h^{-1}, n\right)$ & $\max _{G^{S} \cap\{r \geq 0.2\}} r^{1 / 3}\left\|\partial U_{h}^{\varepsilon} / \partial x-\partial u / \partial x\right\|$ & $\max _{G^{S} \cap\{r \geq 0.2\}} r^{1 / 3}\left\|\partial U_{h}^{\varepsilon} / \partial y-\partial u / \partial y\right\|$ \\
\hline$(16,45)$ & $7.407 \times 10^{-4}$ & $7.407 \times 10^{-4}$ \\
$(32,70)$ & $1.771 \times 10^{-4}$ & $1.772 \times 10^{-4}$ \\
$(64,70)$ & $4.630 \times 10^{-5}$ & $4.630 \times 10^{-5}$ \\
$(128,75)$ & $1.294 \times 10^{-5}$ & $1.294 \times 10^{-5}$ \\
\hline
\end{tabular}

where $\tilde{x}=r^{2 / 3} \cos (2 \theta / 3)$, and $\tilde{y}=r^{2 / 3} \sin (2 \theta / 3)$. Since we have only one singular point, we omitted subindices in (8.5). We calculate the values $Q^{\varepsilon}\left(r_{12}, \theta^{q}\right)$ and $Q^{\varepsilon}(r, \theta)$ on the grids $t_{1}^{h}$, with an accuracy of $\varepsilon$ using the quadrature formulae proposed in [15]. 
Table 5: The stress intensity factor for the pairs $\left(h^{-1}, n\right)$ when $\varepsilon=5 \times 10^{-9}$.

\begin{tabular}{lc}
\hline$\left(h^{-1}, n\right)$ & Stress intensity factor \\
\hline$(16,45)$ & 1.000004963400265 \\
$(16,75)$ & 1.000013397592036 \\
$(32,65)$ & 0.999994989029130 \\
$(32,75)$ & 0.9999973755906025 \\
$(32,90)$ & 0.9999973633441369 \\
$(64,65)$ & 1.000001087887584 \\
$(64,70)$ & 1.000000179113669 \\
$(64,100)$ & 0.9999997881749654 \\
$(128,65)$ & 1.000000056469357 \\
$(128,75)$ & 1.000000202312094 \\
$(128,80)$ & 1.000000008763244 \\
\hline
\end{tabular}

On the basis of (7.5) and (8.5) for the stress intensity factor, we have

$$
\tau_{1, n}^{\varepsilon}=\frac{3}{28 \pi}+\frac{2}{n(0.93)^{2 / 3}} \sum_{q=1}^{n}\left(u_{h}^{\varepsilon}\left(0.93, \theta_{j}^{q}\right)-Q_{j 2}^{q \varepsilon}\right) \sin \frac{2}{3} \theta_{j}^{q} .
$$

Taking the zero approximation $u_{h}^{\varepsilon(0)}=0$, the results of realization of the iteration (6.1) for the solution of the problem in Example 8.1 are given in Tables 1-4. Tables 1 and 2 represent the order of convergence

$$
\mathfrak{R}_{G^{\mathrm{NS}}}^{m}=\frac{\max _{G^{\mathrm{NS}}}\left|u_{2^{-m}}^{\varepsilon}-u\right|}{\max _{G^{\mathrm{NS}}}\left|u_{2^{-(m+1)}}^{\varepsilon}-u\right|}
$$

in "nonsingular", and the order of convergence

$$
\mathfrak{R}_{G^{S}}^{m}=\frac{\max _{G^{s}}\left|U_{2^{-m}}^{\varepsilon}-u\right|}{\max _{G^{S}}\left|U_{2^{-(m+1)}}^{\varepsilon}-u\right|}
$$

in "singular" part of $G$, respectively, for $\varepsilon=5 \times 10^{-9}$. In Table 3, the minimal values over the pairs $\left(h^{-1}, n\right)$, of the errors in maximum norm, of the approximate solution when $\varepsilon=5 \times 10^{-9}$ are presented. The similar values of errors for the first-order derivatives are presented in Table 4 when $\partial Q / \partial x$ and $\partial Q / \partial y$ are approximated by second-order central difference formula on $G^{S}$ for $r \geq 0.2$. For $r<0.2$, the order of errors decrease is down to $10^{-3}$ which are not presented in Table 4. This happens because the integrands in (8.5) are not sufficiently smooth for second-order differentiation formula. The order of accuracy of the derivatives for $r<0.2$ can be increased if we use similar quadrature rules which we used for the integrals in (8.5) for the derivatives of integrands also. Table 5 represents the stress intensity factor for the pairs $\left(h^{-1}, n\right)$ when $\varepsilon=5 \times 10^{-9}$.

Figures 2 and 3 show dependence on $\varepsilon$ for different mesh steps $h$. Figure 4 demonstrates the convergence of BGM with respect to the number of quadrature nodes for 


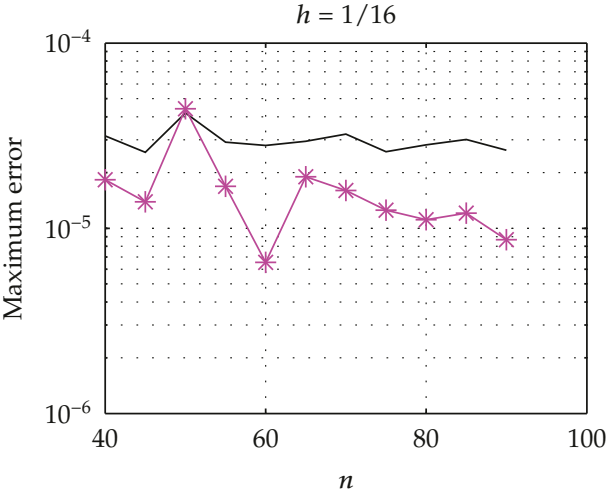

(a)

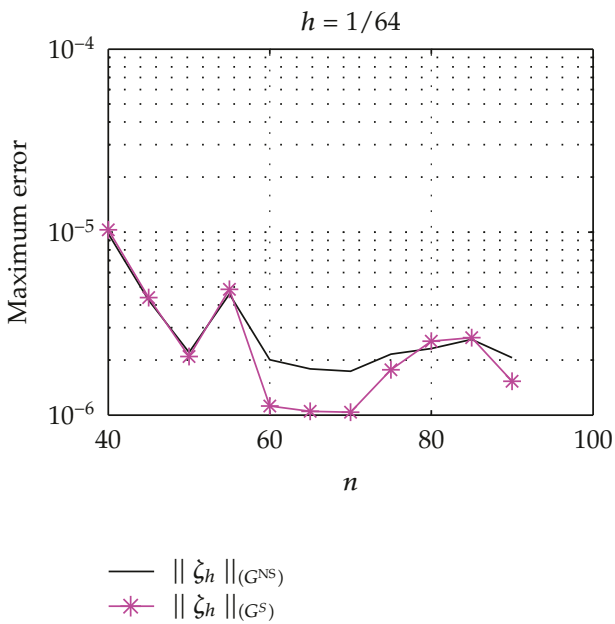

(c)

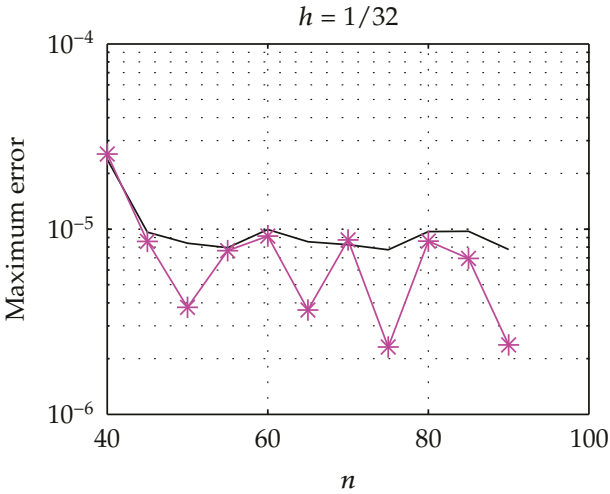

(b)

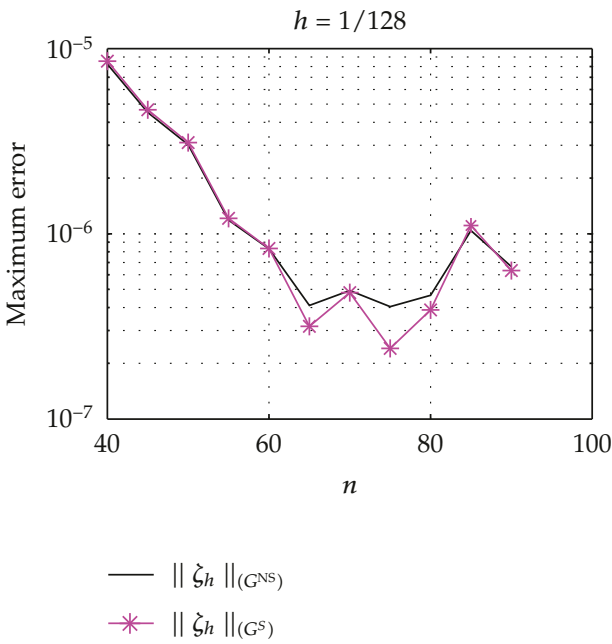

(d)

Figure 4: Maximum error depending on the number of quadrature nodes $n$.

different mesh steps $h$. The approximate solution and the exact solution in "singular" part are given in Figure 5 to illustrate the accuracy of the BGM. The error function for $\varepsilon=5 \times 10^{-9}$ of calculating the function $Q(r, \theta)$ in (8.5) is presented in Figure 6. Figures 7 and 8 show the behavior of the first-order partial derivatives of the approximate solution in "singular" part.

\section{Conclusions}

We have developed the block-grid method for nonanalytic boundary conditions of the first kind on the whole boundary, that is, we remove the restriction on the boundary functions to be algebraic polynomials on the sides of the polygon causing the singular vertices. It is assumed that the boundary function on the whole boundary is given from the Hölder classes $C^{2, \lambda}, 0<\lambda<1$. In the integral representations around each singular vertex, which are combined with the finite difference equations on "nonsingular" part, the boundary conditions are taken into account with the help of integrals of Poisson type for a half-plane. 


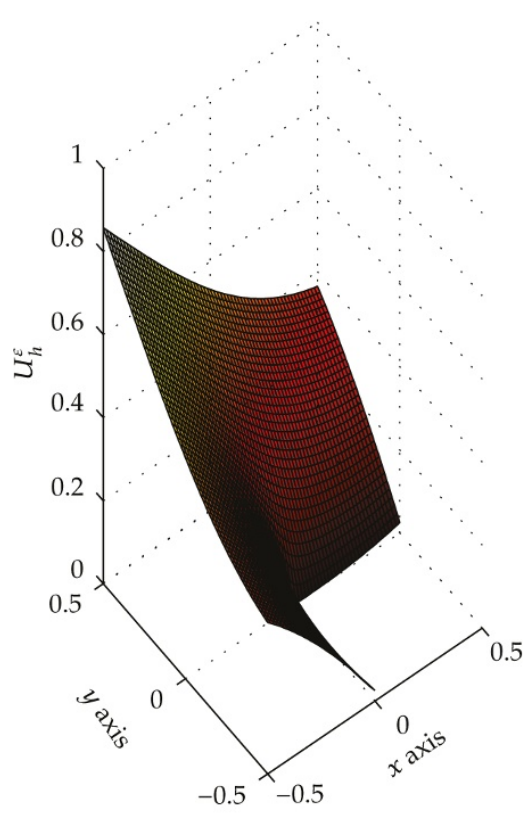

(a)

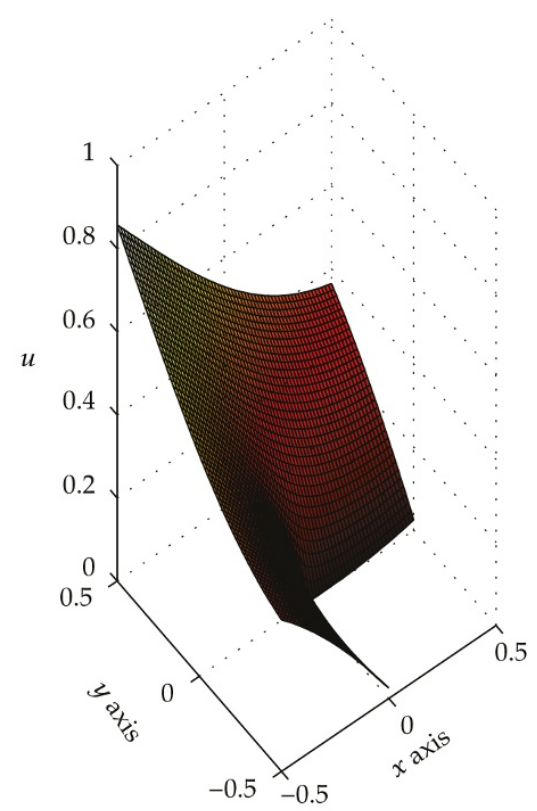

(b)

Figure 5: The approximate solution $U_{h}^{\varepsilon}$ and the exact solution $u$ in the "singular" part for $\varepsilon=5 \times 10^{-9}$.

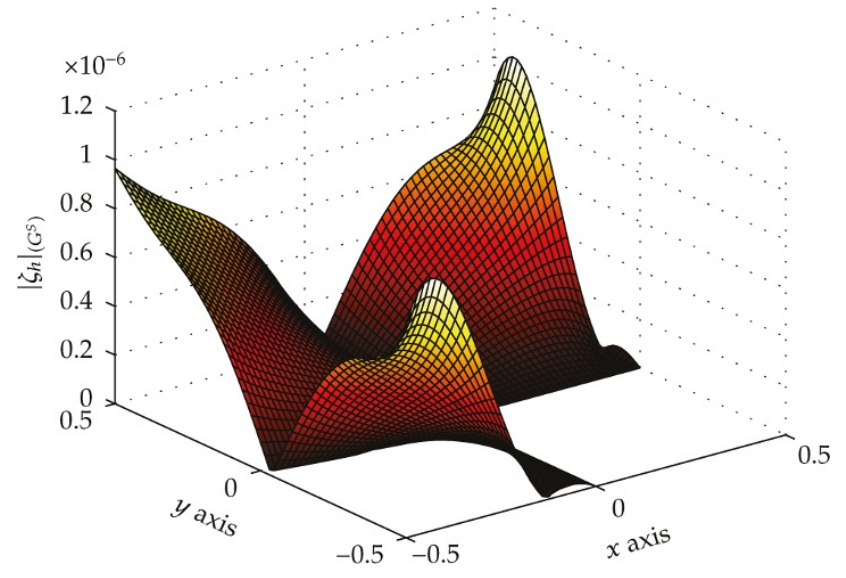

Figure 6: The error function in "singular" part when $\varepsilon=5 \times 10^{-9}$.

It is proved that the final uniform error is of order $O\left(h^{2}+\varepsilon\right)$, where $\varepsilon$ is the error of the approximation of the mentioned integrals, and $h$ is the mesh step. For the $p$-order derivatives $(p=0,1, \ldots)$ of the difference between the approximate and the exact solutions in each "singular" part, $O\left(\left(h^{2}+\varepsilon\right) r_{j}^{1 / \alpha_{j}-p}\right)$ order is obtained. The method is illustrated in solving the problem in L-shaped polygon with the corner singularity, and a simple formula for the stress intensity factor for a high accurate approximation is given. Moreover, dependence of 


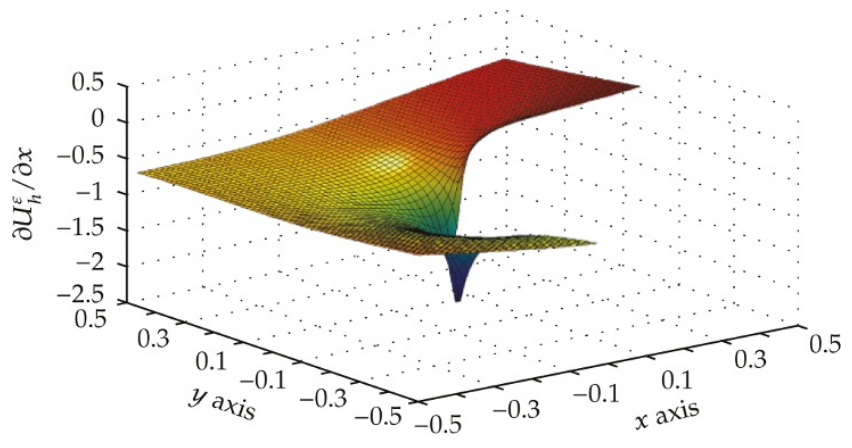

Figure 7: $\partial U_{h}^{\varepsilon} / \partial x$ in the "singular" part.

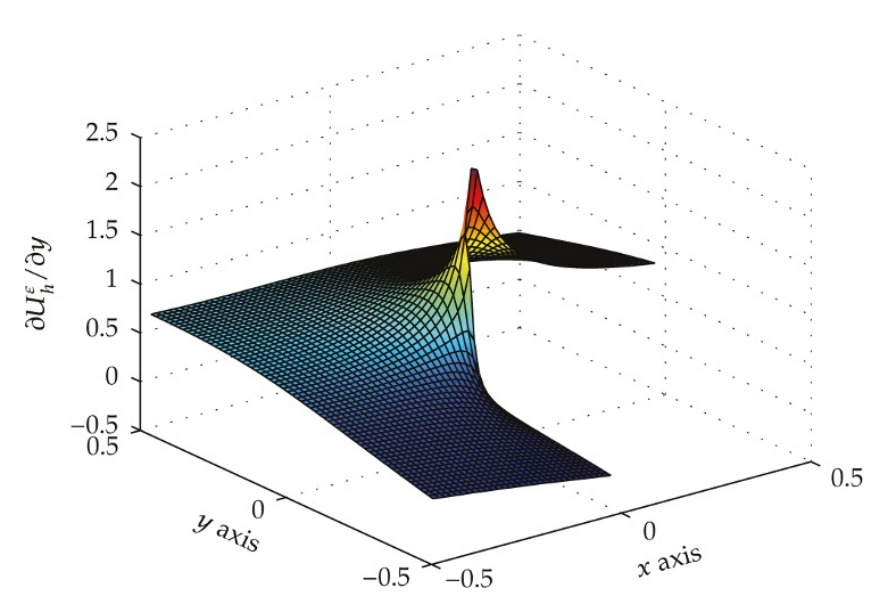

Figure 8: $\partial U_{h}^{\varepsilon} / \partial y$ in the "singular" part.

the approximate solution and its errors on $\varepsilon, h$, and a number of quadrature nodes $n$ are demonstrated.

\section{Acknowledgment}

This work was supported by the Ministry of National Education and Culture of TRNC under project MEKB-09-03.

\section{References}

[1] Z. C. Li, Combined Methods for Elliptic Equations with Singularities, Interfaces and Infinities, vol. 444 of Mathematics and Its Applications, Kluwer Academic Publishers, Dordrecht, The Netherlands, 1998.

[2] Z. C. Li and T. T. Lu, "Singularities and treatments of elliptic boundary value problems," Mathematical and Computer Modelling, vol. 31, no. 8-9, pp. 97-145, 2000.

[3] A. A. Dosiyev, "The high accurate block-grid method for solving Laplace's boundary value problem with singularities," SIAM Journal on Numerical Analysis, vol. 42, no. 1, pp. 153-178, 2004.

[4] A. A. Dosiyev, "A block-grid method of increased accuracy for the solution of the Laplace equation on polygons," Russian Academy of Sciences. Doklady. Mathematics, vol. 45, no. 2, pp. 396-399, 1992. 
[5] A. A. Dosiyev, "A block-grid method of increased accuracy for solving the Dirichlet problem for the Laplace equation in polygons," Computational Mathematics and Mathematical Physics, vol. 34, no. 5, pp. 591-604, 1994.

[6] A. A. Dosiyev and S. Cival, "A difference-analytical method for solving Laplace's boundary value problem with singularities," in Proceedings of Conference on "Dynamical Systems and Applications", pp. 339-360, Antalya, Turkey, July 2004.

[7] A. A. Dosiyev and S. Cival, "A combined method for solving Laplace's boundary value problem with singularities," International Journal of Pure and Applied Mathematics, vol. 21, no. 3, pp. 353-367, 2005.

[8] A. A. Dosiyev and S. C. Buranay, "A fourth order accurate difference-analytical method for solving Laplace's boundary value problem with singularities," in Mathematical Methods in Engineers, K. Tas, J. A. T. Machado, and D. Baleanu, Eds., pp. 167-176, Springer, New York, NY, USA, 2007.

[9] E. A. Volkov, "An exponentially converging method of solving the Laplace equation on polygons," Mathematics of the USSR, Sbornik, vol. 37, no. 3, pp. 295-325, 1980.

[10] E. A. Volkov, Block Method for Solving the Laplace Equation and for Constructing Conformal Mappings, CRC Press, Boca Raton, Fla, USA, 1994.

[11] G. J. Fix, S. Gulati, and G. I. Wakoff, "On the use of singular functions with finite element approximations," Journal of Computational Physics, vol. 13, pp. 209-228, 1973.

[12] V. A. Kondratiev, "Boundary value problems for elliptic equations in domains with conical or angular points," Transactions of the Moscow Mathematical Society, vol. 16, pp. 227-313, 1967.

[13] P. Grisvard, Elliptic Problems in Nonsmooth Domains, vol. 24 of Monographs and Studies in Mathematics, Pitman, Boston, Mass, USA, 1985.

[14] S. Cho and M. Safonov, "Hölder regularity of solutions to second-order elliptic equations in nonsmooth domains," Boundary Value Problems, vol. 2007, Article ID 57928, 24 pages, 2007.

[15] E. A. Volkov, "Approximate solution by the block method of the Laplace equation on polygons with nonanalytic boundary conditions," Proceedings of the Steklov Institute of Mathematics, no. 4, pp. 65-90, 1992.

[16] A. A. Dosiyev, "A fourth order accurate composite grids method for solving Laplace's boundary value problems with singularities," Computational Mathematics and Mathematical Physics, vol. 42, no. 6, pp. 832-849, 2002.

[17] V. P. Mijailov, Partial Differential Equations, Mir, Moscow, Russia, 1978.

[18] E. A. Volkov, "The method of composite regular nets for the Laplace equation on polygons," Trudy Matematicheskogo Instituta imeni V. A. Steklova, vol. 140, pp. 68-102, 1976.

[19] E. A. Volkov, "Approximate solution by the block method of the Laplace equation on polygons with analytic mixed boundary conditions," Proceedings of the Steklov Institute of Mathematics, no. 2, pp. 137153, 1994.

[20] E. A. Volkov, "Differentiability properties of solutions of boundary value problems for the Laplace and Poisson equations on a rectangle," Proceedings of the Steklov Institute of Mathematics, vol. 77, pp. 101-126, 1965. 